\title{
Construction of New Experimental Teaching System of Cognitive Psychology in the Era of Big Data
}

\author{
Zhao Kang ${ }^{1, *}$, Youli Kang ${ }^{2}$ \\ ${ }^{1}$ School of Education, Research Center for Mental Health Education, China West Normal University, Nanchong, China \\ ${ }^{2}$ Psychological Counseling Center for University Students, China West Normal University, Nanchong, China \\ *Corresponding author
}

\begin{abstract}
The highly developed network information technology in the era of big data is bound to bring about great changes in teaching ideas and methods. Cognitive psychology is strongly theoretical and practical, and the traditional experimental teaching system has already not met the basic needs of subject teaching in the current era of big data. The experimental teaching of cognitive psychology in colleges and universities should actively comply with the basic requirements of the era of big data, continuously innovate the teaching objectives, contents, conditions, management and assessment system of the experimental teaching of cognitive psychology, and scientifically construct a new experimental teaching system of cognitive psychology.
\end{abstract}

Keywords: The era of big data, Cognitive psychology, Experimental teaching, Teaching system

\section{大数据时代认知心理学科课程实验教学新体系构建}

\author{
康㻇 $1{ }^{1, *}$, 康有黎 2 \\ ${ }^{1}$ 西华师范大学教育学院, 心理健康教育研究中心, 南充, 中国 \\ 2西华师范大学, 大学生心理咨询中心, 南充, 中国 \\ “通讯作者

\section{中文摘要} \\ 大数据时代高度发达的网络信息技术必然带来教学理念与教学实施方式的重大变革。认知心理学科的 \\ 理论性与实践操作性极强, 传统的实验教学体系已经无法满足当前大数据时代学科教学的基本需要。 \\ 高校认知心理学科实验教学应当积极顺应大数据时代的基本要求, 不断创新认知心理学科实验教学目 \\ 标、内容、条件、管理与评价体系, 科学构建认知心理学科实验教学新体系。 \\ 关键词: 大数据时代; 认知心理学; 实验教学; 教学体系
}

\section{1. 引言}

认知心理学是现代心理学科体系中极其重要的分 支学科, 在当前心理学科研究体系和相关领域中表现十 分活跃。认知心理学是我校心理学及教育类相关专业本 科生的专业必修课程, 在大三阶段第五学期开出本门课 程。学科教学过程中既强调学科基本理论知识的系统讲 解, 更重视学生实验实践教学活动的有效开展; 既强调 认知心理学实验原理知识的系统学习, 也强调学生独立 设计实验等综合实践能力的培养。特别是当前认知心理 科学发展日新月异, 重视学科综合性实验设计成为学科
教学发展的必然趋势, 开发和设计前沿性、交叉性和应 用性并重的学科综合实验已经成为认知心理学实验教 学改革的重要任务 [1]。当前正处于大数据时代, 传统 的实验教学体系已经无法满足认知心理学科课程教学 的基本要求。我们必须科学把握大数据时代的核心特征, 积极构建基于大数据时代的认知心理学科实验教学新 体系, 促进认知心理学科教学体系的不断完善与发展。 


\section{2. 大数据时代对认知心理学科实验教学体系 提出的新要求}

随着我国科学技术的不断发展和进步, 互联网络领 域的丰硕成果已逐步渗透并拓展到教学领域之中, 大数 据逐渐受到越来越多研究者和使用者的关注。大数据意 即海量的信息资料, 是指由数量巨大、结构复杂、类型 众多的数据所构成的数据集合体, 其核心的价值在于通 过对海量数据资源进行有效存储和分析处理以更好服 务于人类社会 $[2]$ 。大数据时代高度发达的信息技术带 来教学理念、教学结构与教学实施方式等方面的重大转 向与变革。大数据要求教学与信息技术的高度融合, 由 此促使教学逐渐走向科学化、个性化与多元化的发展道 路。大数据给教学所带来的积极影响正逐渐显露头角, 数据驱动的教学模式已然成为现代教学的主流趋势, 并 不断引领教学改革走向深入 [3]。大数据时代对当前认 知心理学科实验教学体系同样提出了更新更高的要求。

大数据时代下认知心理学科实验教学信息数据体 量极其庞大, 实验教学信息数据的获取更加快捷和完整, 教师在教学过程中可以更加高效地借助海量信息资源, 充分实现课堂实验教学的价值引领, 科学利用大数据带 来的各种优势促进学科实验教学的全新变革, 更好地针 对学生的实际状况来进行学科实验教学。大数据时代认 知心理学科实验教学资源极为丰富, 实验教学形式也不 再满足于教师简单讲授学科基本理论知识、学生机械重 复一些验证性实验等极其传统僵化的操作流程。同时, 大数据时代各种网络在线教育平台的全面介入使认知 心理学科实验教学过程同样可能变得更加生动形象, 诸 如微课、慕课、虚拟课堂等教学资源的积极推进使得认 知心理学科实验教学内容更加丰富多彩, 实验教学形式 变得灵活多样, 学生可以借助各种实验教学平台充分实 现自主学习。随着信息技术的不断发展, 认知心理学科 实验教学载体、内容、形态同样呈现多样化发展态势, 各类音视频和虚拟实验教学资源极其丰富, 多媒体课件、 实物投影仪以及应用 APP 逐渐演变成新的实验教学媒介 [4]。学生能够积极参与并体验知识的发现过程, 使知 识获取的过程与实验实践操作能够充分呈现在具体的 实验实践教学情景之下。因此, 大数据时代对认知心理 学科教师的实验实践教学理念、教学管理、教学方式与 教学评价等不可避免地会产生诸多影响, 我们必须重视 互联网大数据时代下教师实验实践教学理念的全新塑 造, 注重互联网大数据时代认知心理学科实验教学体系 的实践探索, 更好地服务于认知心理学科实验教学工作。

\section{3. 积极构建适应大数据时代要求的认知心理 学科实验教学新体系}

\section{1. 重视认知心理学科实验教学目标体系的 创新}

传统的认知心理学课程更多看重对基础性实验的 简单验证, 这种相对单一的教学目标难以适应当前认知 心理学科快速发展态势下的教育需求。当前的认知心理 学课程实验教学应该以大数据时代为背景, 结合认知心 理学科发展状况和行业前沿信息, 探索高性能计算技术、 云技术以及大数据分析构成的实验教学体系, 构建针对 师生主体需求的多层目标体系 [5]。教师应当重视通过 书本、网络等各种途径不断提升自身的专业知识水平、 专业素养和专业技能, 尤其要充分利用网络中海量学科 信息数据, 不断完善学科教学理论体系。同时, 应当重 视引导学生通过网络获取丰富的知识, 开阔学生的眼界, 帮助学生加深对所学知识的理解, 指导学生学会在实践 中不断修正完善和创新知识。在教学实践层面上, 教师 要积极创造条件, 配合学校不断新增或改善实验教学条 件, 努力将学科前沿设备运用于日常教学和科研工作, 帮助学生形成基本的数据分析与处理能力, 学会根据特 定目标进行数据的收集篮选与存储、数据算法分析与预 测、数据分析与结果呈现, 对于全面提升学生的专业实 验实践能力大有助益。

\section{2. 重视认知心理学科实验教学内容体系的 创新}

基于大数据时代要求的新型实验实践教学模式除 了目标体系要有所创新之外, 更应当重视教学内容的创 新发展。认知心理学区别于其他学科的重要特点之一, 在于其极强的实验实践特性。认知心理学科教学内容随 着时代的发展而不断丰富, 学科实验教学内容体系同样 应当随之而作出相应的变化。大数据并不仅仅只是意味 着数据资源的体量十分庞大，同时更预示着可以凭借和 利用多种类型的信息数据获取更加丰富的课程资源, 以 已有的数据作为基础进行深层次的研究并做出更加科 学的决策和利用。大数据处理涉及数据采集、数据存储、 数据分析以及数据可视化等环节, 涉及计算机科学、统 计学和数据挖掘等前沿技术和方法, 认知心理学课程实 验实践教学应该摆脱传统的章节教学和教材体系, 积极 构建基于实际需求的新型教学模块和内容体系 $[6]$ 。大 数据时代高度发达的网络已经成为学生获取知识、了解 世界的重要途径, 教师要积极为学生理论知识的提升奠 定坚实的内容基础。 


\section{3. 重视认知心理学科实验教学条件体系的 创新}

实验教学条件体系的创新同样应当是大数据时代 对认知心理学科新型实验实践教学模式的现实诉求。为 保证大数据时代实验教学工作的正常运行, 实践中应当 重视实验教学资源信息平台建设力度, 特别是加强大数 据教学管理平台、学习与云实验平台等大数据支撑平台 方面的建设, 并可尝试与其他院校共同开发认知心理学 科课程实验实践教学标准和考核标准, 与其他院校和部 门合作开发网络实验教材、实验教学课件与视频资料以 及实验教学技能考核库。同时, 要积极建设高素质的认 知心理学科教师队伍, 科学引领认知心理学科实验实践 教学工作。实践中我们充分根据学生的个体特点和实际 需要, 建立本科学生导师制, 全面开放学院心理实验室, 指导学生学习前沿心理学科设备资源的操作使用, 为日 后开展系统的科学研究奠定坚实的基础。同时, 实践中 我们科学利用各类网络在线课程, 包括虚拟实验教学平 台的推广使用, 在大数据时代下网络课堂在教育领域已 经得到广泛利用并取得积极成效, 已然成为当前实体课 堂教学形式的有益补充。总之, 我们应当积极巩固已有 的实验实践场地，不断拓展和充分利用浩瀚的网络资源 平台作为重要的实验实践基地, 促进认知心理学科线上 实验教学与线下实验实践的紧密结合 [7]。

\section{4. 重视认知心理学科实验教学管理体系的 创新}

基于大数据时代的认知心理学科实验实践教学新 模式, 不仅要在实验教学目标、内容等方面有所创新, 更要形成借助于网络数据的实验教学管理体系。随着时 代的发展变迁, 传统的数据管理和硬件基础架构的管理 模式难以支撑大数据实验室不断发展的高标准要求, 为 全面提升学生的学习成效, 实践中我们尝试采用打破专 业设置的界限, 将不同专业学生集中起来进行学科实验 实践能力的综合训练, 采用自主式、团队式、研究式等 多种学习模式有机结合, 将学校心理学、教育学、教育 技术学等专业学生中间对认知心理学科实验教学极感 兴趣的同学组织起来成立认知心理学科研究小组, 充分 利用课余时间强化认知心理学科实验实践教学技能训 练, 也就是尝试进行认知心理学科跨专业综合实践教学 模式的有益探索, 取得了较为满意的成效, 部分学生还 因此成功申请到行业领域的创新创业项目。同时, 大数 据作为认知心理学科新兴技术和学科发展方向, 学科实 验教学缺少相应的实验教学环境和平台是困扰学科发 展的重大问题。因此, 应当重视基于网络大数据要求积 极建设统一的学科实验教学与管理平台, 对认知心理学 科实验实践教学情况进行监督和不定期检查, 对其实施 情况、教学内容等各方面做出全面估量, 对教师的实验 教学成果、学生实验实践操作情况进行监督和评价。针 对大数据技术认知心理学课程实验教学的特点和学生 培养目标, 研究相关背景下教学实验环境的积极建设工
作，加深师生对大数据技术理论知识的理解和掌握，帮 助教师有效利用大数据技术更好地服务于认知心理学 科实验教学以及由此促进学生实验操作技能的不断提 升。

\section{5. 重视认知心理学科实验教学评价体系的 创新}

从一定程度上说, 建立较为科学完善的实验教学评 价体系是决定认知心理学科实验教学水平提升的关键 因素。实践中我们结合大数据时代对实验教学的新要求, 改变以往认知心理学课程过分看重实验教学结果忽视 过程的教学评价方法, 并从认知心理学科课程在时间结 构上的开放性、综合性、实践性的特点出发，将基础性 实验、综合设计类实验、研究创新类实验有机结合，把 实验教学与科技竞赛、创新创业项目有机结合, 致力于 构建一种最终考核与阶段考核相结合、更加注重阶段性 考核的教学评价体系 [8]。同时，改变传统实验教学的 单一考核方法, 既重视理论学习考核, 更强调实验实践 操作和实验实践研究报告的撰写, 以及参与社会实践活 动方面的成果考核等灵活多样的考核方法, 更加关注学 生的学习能力和智慧能力的获取, 或者说更加关注教学 活动对师生创造能力促进方面的评价, 更加关注教学对 学生创新能力提供的贡献力。

\section{4. 结语}

可以说, 站在以互联网大数据作为代表的时代潮流 面前, 基于大数据时代特征积极进行认知心理学科实验 教学新体系建设的实践探索, 促进认知心理学科实验教 学体系更加科学准确且富有实效, 自然能够充分满足大 数据时代广大师生对认知心理学科实验教学的实际需 要，并不断提升认知心理科学教学整体质量。

\section{致谢}

西华师范大学校级教学改革与研究项目: 认知心理 学科跨专业综合实验教学体系建设研究 (xjjgxh2017061)。

\section{REFERENCES}

[1] Kang Zhao, Zhou Zhihui. Discussion on Teaching Mode of Trans-Disciplinary Comprehensive Experiment in Cognitive Psychology. Journal of Higher Education, No.19, pp.139-141, 2018.

[2] Zhang Yang. Big data to Improve the Quality and Management of Experimental Teaching in Colleges and Universities, Modern Measurement and Laboratory Management, No.5, pp.68-70, 2016. 
[3] Yang Xianmin, Luo Jiaojiao, Liu Yaxin, Chen Shichao. Data-driven Instruction: A New Trend of Teaching Paradigm in Big Data Era. E-education Research, No.12, pp.13-20, 2017.

[4] Yang Ruina. New Paradigm of College Teaching in the Era of Big Data. China Computer \& Communication, No.15, pp.231-232, 2019.

[5] Li Hongjian. Research on Experimental Teaching Reformation for Computer Majors in the Era of Big Data. Modern Computer, No.19, pp.29-31, 2013.

[6] Gao Mingyue, Pan Zhenggao. Research and Design of Experimental Teaching System for Big Data Major, Journal of Ezhou University, No.2, pp.107-109, 2019.

[7] Duan Ruiqin, Zhao Changxing. The New Mode Construction of Curriculum Practice Teaching System in the Age of Big Data, Ship Building Vocational Education, No.4, pp.55-58, 2015.

[8] Wang Fang, Yang Lei, Guo Huiting, Ji Jingying. Exploration of Experiment Teaching Reform for Economic and Management Specialties under the Background of Big Data, Laboratory Science, No.3, pp.72-75, 2016. 